\title{
Clinical utility of ultrasonographic evaluation in acute kidney injury
}

\author{
Caijie Liu, Xiuzhen Wang \\ Department of Ultrasound, First Affiliated Hospital of Jinzhou Medical University, Jinzhou 121001, China \\ Contributions: (I) Conception and design: C Liu; (II) Administrative support: X Wang; (III) Provision of study materials or patients: C Liu; (IV) \\ Collection and assembly of data: C Liu; (V) Data analysis and interpretation: C Liu, X Wang; (VI) Manuscript writing: All authors; (VII) Final \\ approval of manuscript: All authors. \\ Correspondence to: Xiuzhen Wang. Department of Ultrasound, First Affiliated Hospital of Jinzhou Medical University, Jinzhou 121001, China. \\ Email: wxz1979jz@126.com.
}

Background: This study aimed to evaluate ultrasonography (US) in patients with acute kidney injury (AKI) and the association of US findings with its clinical characteristics.

Methods: This single-center retrospective study evaluated US in AKI patients. A healthy control group was matched by sex and age at a ratio of 2:1 with the AKI group. The US characteristics were compared between the two groups.

Results: The US characteristics of 111 patients with AKI were evaluated. Compared with the control group, AKI patients had greater kidney length and kidney volume $(\mathrm{P}<0.05)$. Patients with AKI also displayed thicker parenchyma than those in the control group, but only the difference in the right kidney was found to be significant. Of the 111 AKI patients, 38 had positive US findings including increased parenchymal echogenicity, increased renal resistance index (RRI), and hydronephrosis, while only 5 patients had increased RRI. The cause of AKI was attributed to obstructive nephropathy in eight patients.

Conclusions: Although US evaluation indicated that most of the patients with AKI were "normal ultrasound imaging", abnormal findings beyond obstructive nephropathy were still detected in some cases. Aside from its ability to exclude obstructive nephropathy, US evaluation might hold further value. It was found that the kidney size of AKI patients is significantly larger than that of healthy controls. Kidney size combined with other ultrasound indicators could hold potential for the evaluation of AKI.

Keywords: Acute kidney injury (AKI); ultrasonography (US); clinical characteristics; parenchymal echogenicity; renal resistance index (RRI)

Submitted Feb 11, 2020. Accepted for publication May 26, 2020.

doi: 10.21037/tau-20-831

View this article at: http://dx.doi.org/10.21037/tau-20-831

\section{Introduction}

The renal cortex, medulla, and collecting system have different acoustic characteristics and can be easily discerned by ultrasonography (US) (1). US is particularly useful for the evaluation of kidney diseases (1). Previous studies have indicated that renal histopathological changes were correlated and sonographic findings (2-4). Computed $\mathrm{X}$-ray tomography (CT) has higher sensitivity than US in the detection of neoplasms, stones, and calcifications (1). Meanwhile, magnetic resonance imaging (MRI) holds the unique advantage of being able to provide better visualization of the blood vessels (1). However, neither CT nor MRI have any proven advantages over US in the evaluation of kidney failure (1).

Considering its safety, simplicity, and low cost, US has long been regarded as the first-choice imaging method for examining nephrology patients (1). However, renal US is not considered to be essential for patients with acute kidney injury (AKI), and it is typically only used so that post-renal obstruction can be excluded $(1,5)$. Furthermore, the use of renal US for AKI patients even for this reason is uncommon $(5,6)$, and most cases of obstructive nephropathy can be easily recognized because of its clinical characteristics $(5,6)$. 
US was found in previous studies to yield no useful findings and lead to no changes in the clinical management of most AKI patients $(5,6)$. Traditional grayscale ultrasound is lack of sensibility and specificity in identifying and evaluating AKI) (7). It is for these reasons that renal US is not used for routine evaluation of AKI $(1,5,6)$.

Compared with gray-scale ultrasound, Doppler US of the kidney has a limited role in evaluating AKI (1). During diagnostic workup, AKI may be attributed to a variety of different etiologies, including volume depletion, ATN, heart failure, sepsis, or urinary tract obstruction. The coexistence of chronic kidney disease (CKD) and AKI can lead to more complex changes in the acoustic characteristics (1). However, combination of the grayscale ultrasound and echo-color Doppler as a tool in determining intrarenal parenchymal arteries in the for differential diagnosis and predicting for clinical outcomes is useful $(7,8)$. Renal resistance index (RRI) as assessed by Doppler US is considered to be a potential predictor of AKI $(7,8)$. Although US is useful for evaluating the kidney, its value for evaluating AKI that is not caused by an obstruction remains unclear. It has been reported that patients who survived after AKI are predisposed to chronic kidney disease (CKD) (9). US evaluation could provide more detailed information that can influence clinical management and improve the long-term outcome in subtle ways (1). The potential value of US in evaluating AKI requires further investigation. This study aimed to describe the use of US in evaluating AKI and the association between US findings and its clinical characteristics.

\section{Methods}

\section{Ethical approval}

This study strictly adhered to the guidelines of the Declaration of Helsinki of the World Medical Association. This retrospective study was approved by the ethics committee of The First affiliated Hospital of Jinzhou Medical University (File number: 202003). The ethics committee of the hospital waived the need for written informed consent from the patients due to the study's retrospective nature.

\section{Inclusion and exclusion criteria}

Discharged AKI cases from between January 1, 2019 and December 31, 2019 from the inpatient database were screened, and a retrospective review of the patients' medical records was carried out. The inclusion criteria were as follows: (I) a confirmed diagnosis of AKI, which was defined as any of the following criteria: increase in serum creatinine by $\geq 0.3 \mathrm{mg} / \mathrm{dL}(\geq 26.5 \mu \mathrm{mol} / \mathrm{L})$ within 48 hours; or increase in serum creatinine of $\geq 1.5$ times from baseline which is known or presumed to have occurred within the 7 days prior (10); (II) US was performed after AKI onset; (III) an intervention to relieve the obstruction was not performed before US evaluation; and (VI) serum creatinine levels had not recovered before US evaluation. Patients aged $<18$ years or those who were pregnant or 6 weeks postpartum were excluded.

After the AKI cases were screened, the healthy controls were randomly selected by matching sex and age at a ratio of 2:1 with those in the AKI group. The inclusion criteria of the control group were as follows: (I) sex and age matched with the AKI group; (II) renal US kidney evaluation was performed at the hospital between January 1, 2019 and December 31, 2019;

The exclusion criteria of the control group were as follows: (I) individuals aged $<18$ years; (II) subjects who were pregnant or 6 weeks postpartum ; (III) levels of serum creatinine $>103 \mu \mathrm{mol} / \mathrm{L}$; (IV) urinalysis indicated proteinuria or microscopic hematuria; (V) CKD history; (VI) renal US evaluation indicated the presence of renal cysts, hydronephrosis, urinary retention, kidney mass, or other abnormalities; (VII) US evaluation indicated increased echogenicity in renal parenchyma.

\section{Data and variables}

Using the inpatient database from January 1, 2019 to December 31, 2019, all cases with acute renal failure, acute kidney injury, acute renal insufficiency, and multiple organ dysfunction syndrome (MODS) were screened. The detailed electronic medical records confirming the diagnosis of AKI and the collected information were reviewed. All US evaluations were also recorded and stored electronically.

Patient data including name, gender, age, primary diseases, possible etiologies of AKI, the highest serum creatinine levels in AKI, and serum creatinine levels after therapy were collected. The US evaluations included: kidney size (the length, width, and thickness of each kidney), the thickness of the renal parenchyma, parenchymal echogenicity, RRI, and other abnormalities (including calculus, nodules, cysts, stones, urinary retention, and hydronephrosis). Hepatic echogenicity was also recorded if present. 


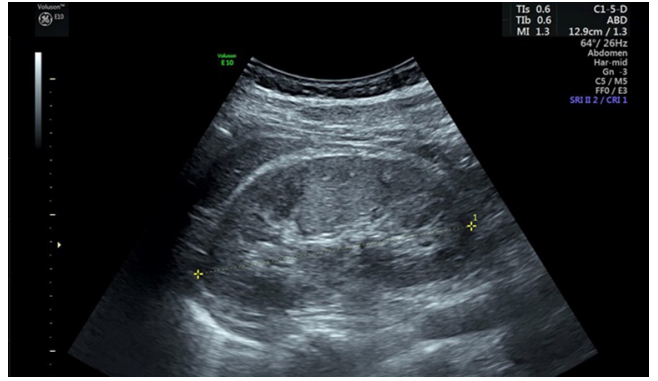

Figure 1 The kidney length was measured as the maximum longitudinal dimension.

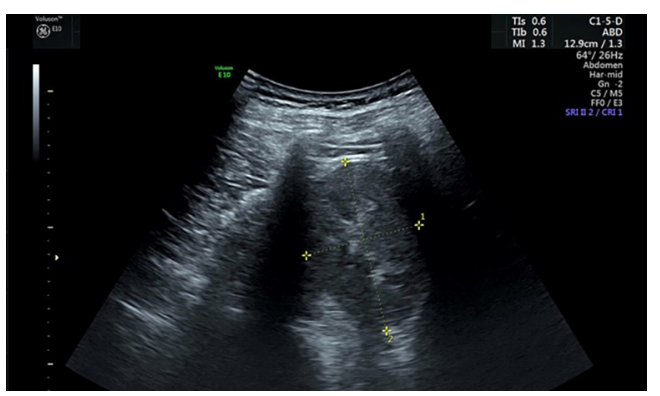

Figure 2 The width and thickness of the kidneys were measured in a section perpendicular to the longitudinal axis of the kidney.

\section{Renal ultrasonography (US)}

Renal US was performed with an Evolution E10 scanner using a 3.5-5.0 MHz wide-frequency band. The US procedures were carried out in our hospital by attending radiologists who had completed thorough training. Two radiologists, including at least one experienced radiologist, interpreted the US images. The parameters measured included the length, width, and thickness of the kidneys. The kidney length was measured as the maximum longitudinal dimension (Figure 1) $(1,4)$. The width and thickness of the kidneys were measured in a section perpendicular to the longitudinal axis of the kidney (Figure 2) (11). The thickness of the parenchyma was determined as the shortest distance from the renal sinus fat to the renal capsule (Figure 3) (4). Kidney volume was determined based on the following formula: Volume $=0.49$ $\times$ kidney length $\times$ kidney width $\times$ kidney thickness (11). The echogenicity of the renal cortex has been found to be lower than the hepatic echogenicity in $90 \%$ of healthy individuals $>7$ years (12), while a minority of healthy individuals have equal cortical and hepatic echogenicity

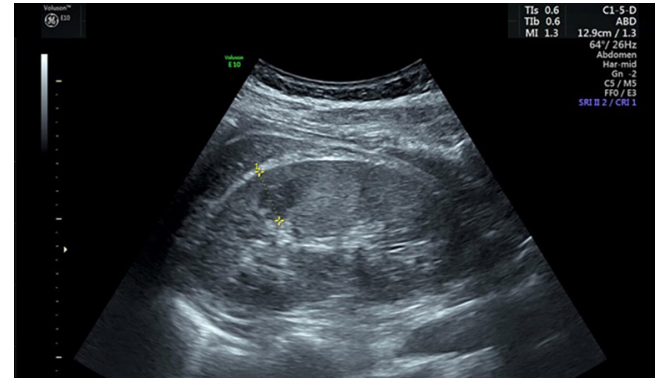

Figure 3 The thickness of the parenchyma was determined as the shortest distance from the renal sinus fat to the renal capsule.

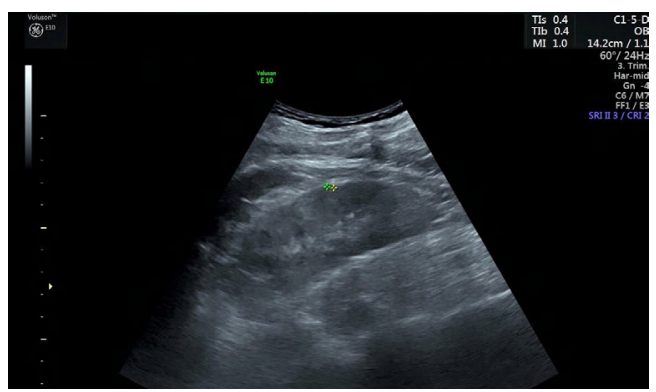

Figure 4 An increase in parenchymal echogenicity was defined as when renal parenchyma was more echogenic than the liver or the spleen.

$(11,13)$. In the present study, parenchymal echogenicity was carefully compared with hepatic echogenicity or splenic echogenicity. The presence of cirrhosis, steatosis, and other diseases of the liver can result in changes in echogenicity, and when these conditions occur, the liver is not considered as a good reference (1). In our study, when the liver showed pathological changes, the spleen was taken as an alternative reference. In the present study, an increase in parenchymal echogenicity was defined as when renal parenchyma was more echogenic than the liver or the spleen (Figure 4).

The RRI was derived from the Doppler spectrum of intrarenal (segmental interlobar) arteries (14). The maximum and minimum (end-diastolic) flow velocities were measured (Figure 5). The RRI was calculated using the following formula: $\mathrm{RRI}=$ (maximum velocity - minimum velocity) maximum velocity (15).

\section{Data analysis}

Data analysis was performed with Stata (version 11). Continuous variables with normal distribution are presented as means \pm standard deviation. Student's $t$-test 


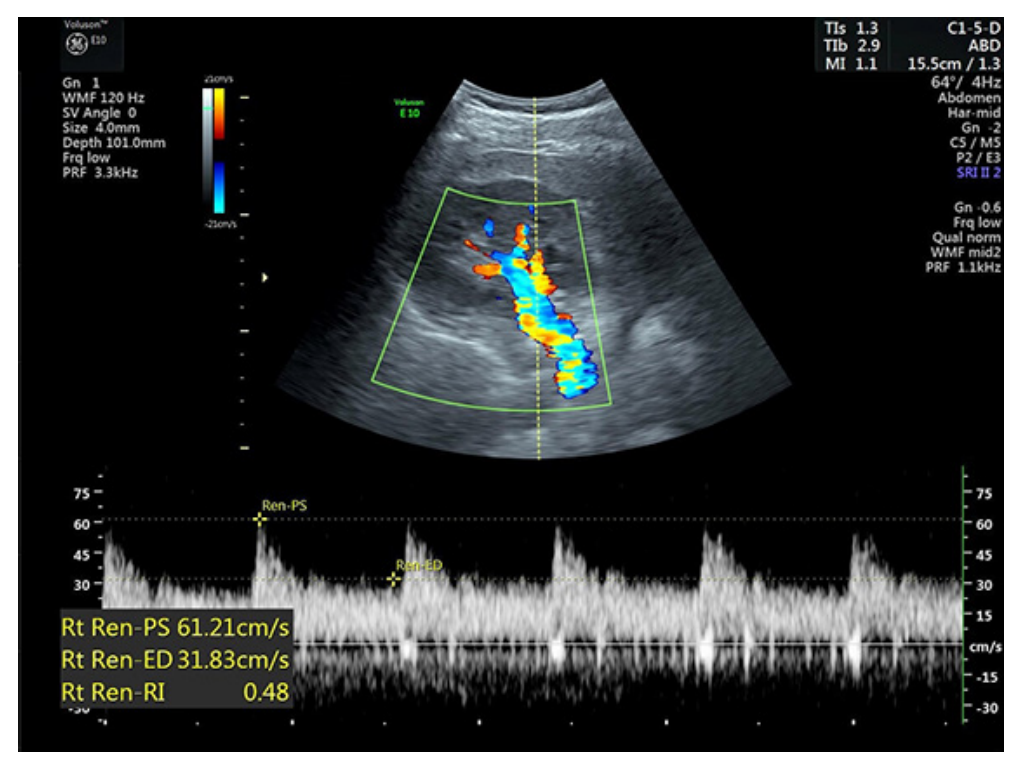

Figure 5 The maximum and minimum (end-diastolic) flow velocities were measured.

was performed to compare the parameters of the AKI group with those of the control group. $\mathrm{P}<0.05$ was considered to be statistically significant difference.

\section{Results}

Between January 1, 2019 and December 31, 2019, 251 cases with acute renal failure, acute renal insufficiency, AKI or MODS during hospitalization were diagnosed at our hospital. Overall, 256 patients with AKI were screened. After repeated cases, pregnant and puerperal women, patients $<18$ years, and patients with chronic renal failure were excluded, there were 226 patients eligible for evaluation. The study included 115 patients with complete US data (Figure S1), and 222 healthy individuals comprised the control group.

\section{The characteristics of 111 patients with AKI (Table 1)}

The data of 111 AKI patients, with a mean age of 59 (range, 18-93) years, were analyzed. Among these 111 patients, $83(74.75 \%)$ were male and $28(25.23 \%)$ were female. The average value of the highest serum creatinine level was $379 \mu \mathrm{mol} / \mathrm{L}$. The etiologies of AKI included volume depletion, heart failure, sepsis, obstructive nephropathy, and ATN (Table 1). In 33 cases, AKI coexisted with glomerular disease, including IgA nephropathy (IgAN), focal segmental glomerulosclerosis (FSGS), lupus nephritis
(LN), membranous nephropathy (MN), antineutrophil cytoplasmic antibody associated vasculitis (AAV), nephrotic syndrome (NS), capillary proliferative glomerulonephritis, post-infectious glomerulonephritis (PIGN), and diabetic nephropathy (DN). In some cases, AKI was caused by multiple etiologies.

\section{Comparison of renal size, parenchymal thickness, and renal volume between the AKI group and control group} (Table 2)

In both the AKI and control groups, the right kidney was slightly smaller in size than the left kidney. Kidney length, width, thickness, and volume in the AKI group were greater than those in the control group $(\mathrm{P}<0.05)$. In the AKI group, the volume of the left kidney was $146.36 \mathrm{~cm}^{3}$, while the volume of the right was $141.48 \mathrm{~cm}^{3}$. The AKI group also had a thicker renal parenchyma than the control group, but the difference was significant in the right kidney. Figure 6 indicated an enlarged kidney and volume increase in AKI.

\section{Abnormal findings of US evaluation in patients with AKI (Table 3)}

In the AKI group, 15 patients had unilateral or bilateral kidneys measuring $>120 \mathrm{~mm}$. The largest kidney size $(143 \mathrm{~mm})$ was a result of unilateral hydronephrosis with infection. In 8 patients, at least one kidney measured 
Table 1 The characteristics of 111 patients with AKI

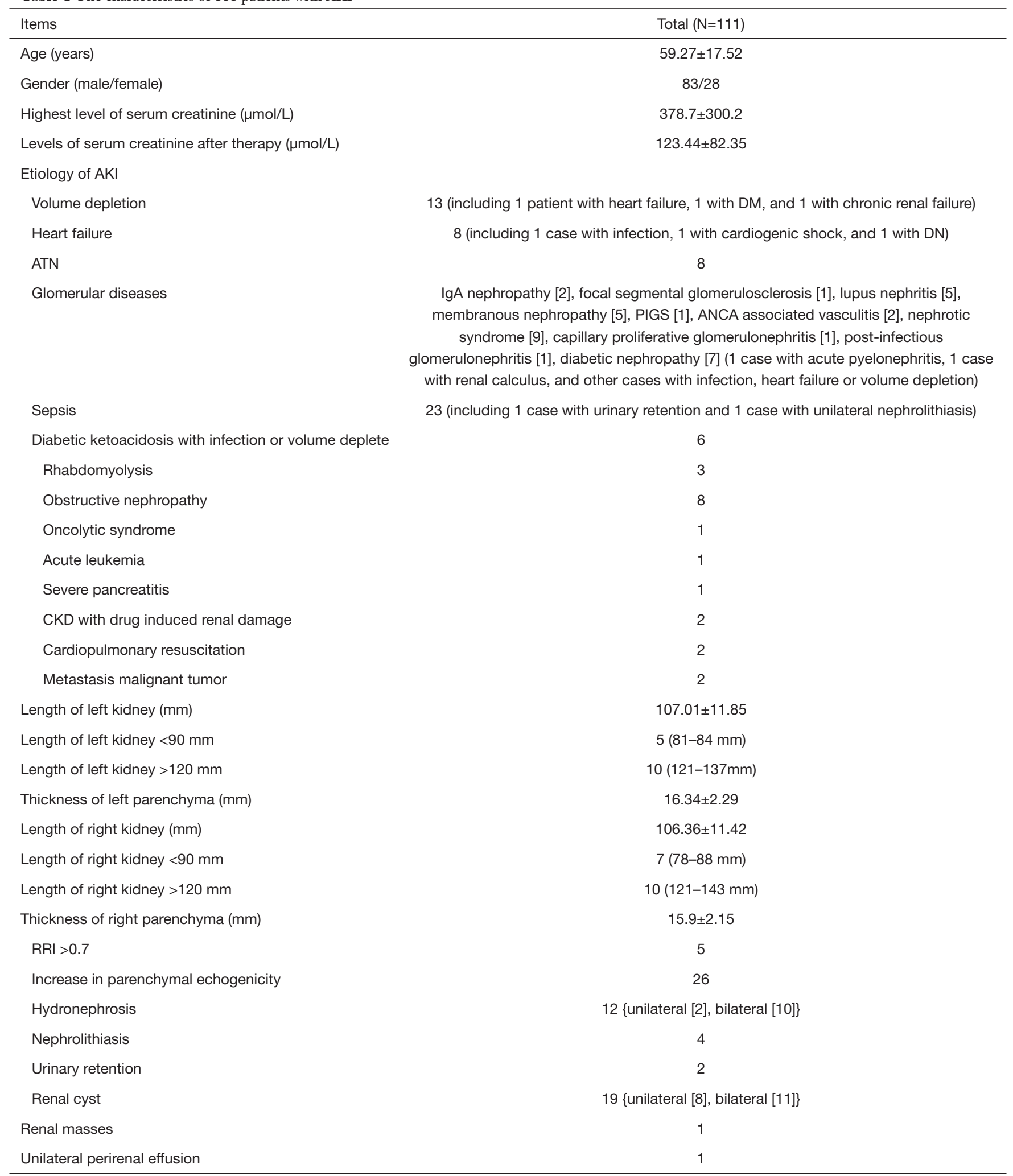

AKI, acute kidney injury; DM, diabetes mellitus; DN, diabetic nephropathy; ATN, acute tubular necrosis; CKD, chronic kidney disease; RRI, renal resistance index. 
Table 2 Comparison of renal size, parenchymal thickness, and renal volume between the AKI group and the control group

\begin{tabular}{|c|c|c|c|}
\hline Items & AKI group $(\mathrm{N}=111)$ & Control group ( $\mathrm{N}=222)$ & $P$ value \\
\hline Gender (male/female) & $83 / 28$ & $166 / 56$ & \\
\hline The level of serum creatinine $(\mu \mathrm{mol} / \mathrm{L})$ & $378.7 \pm 300.2$ & $89.49 \pm 29.34$ & $<0.001$ \\
\hline Length of left kidney (mm) & $107.01 \pm 11.85$ & $104.01 \pm 7.44$ & $<0.05$ \\
\hline Thickness of left kidney (mm) & $48.22 \pm 6.9$ & $45.54 \pm 5.01$ & $<0.05$ \\
\hline Volume of left kidney $\left(\mathrm{cm}^{3}\right)$ & $146.36 \pm 48.66$ & $115.41 \pm 24.63$ & $<0.05$ \\
\hline Thickness of left parenchyma & $16.34 \pm 2.29$ & $16.00 \pm 2.16$ & 0.19 \\
\hline Length of right kidney (mm) & $106.36 \pm 11.42$ & $101.75 \pm 10.49$ & $<0.05$ \\
\hline Volume of right kidney $\left(\mathrm{cm}^{3}\right)$ & $141.48 \pm 46.01$ & $108.26 \pm 28.31$ & $<0.05$ \\
\hline Thickness of right parenchyma & $15.9 \pm 2.15$ & $15.4 \pm 2.10$ & 0.04 \\
\hline
\end{tabular}

AKI, acute kidney injury.

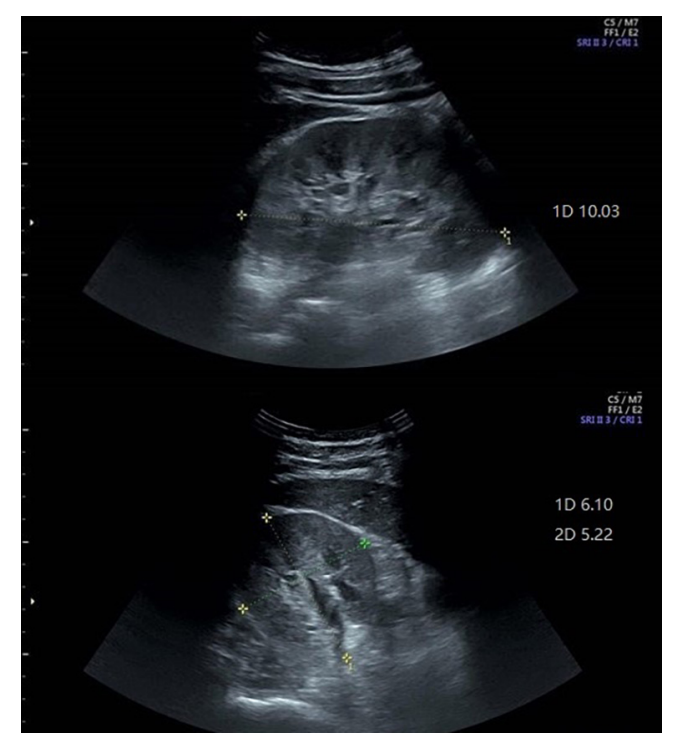

Figure 6 An enlarged kidney. Volume $=0.49 \times 10.03 \mathrm{~cm} \times 6.10 \mathrm{~cm}$ $\times 5.22 \mathrm{~cm}=156.49 \mathrm{~cm}^{3}$.

$<90 \mathrm{~mm}$ in length. Among these 8 patients, the levels of serum creatinine reduced to a normal level after recovery in 7 patients. One patient with CKD had a higher level of baseline serum creatinine $(239 \mu \mathrm{mol} / \mathrm{L})$. The minimum length of the kidney was $78 \mathrm{~mm}$ with a normal baseline serum creatinine level $(84 \mu \mathrm{mol} / \mathrm{L})$.

With the exception of kidney size, 38 of 111 AKI patients had abnormal findings by US evaluation, which included increased parenchymal echogenicity, increased RRI, and hydronephrosis. The most common cause for increased parenchymal echogenicity in AKI patients was glomerular disease. Among the 33 AKI cases with coexisting glomerular diseases, 11 had increased renal parenchymal echogenicity. The main causes of parenchymal echogenicity were: ATN, diabetic ketoacidosis (DKA) with acute pyelonephritis, sepsis, AKI based on chronic renal failure, heart failure, volume depletion, and obstructive nephropathy. However, when parenchymal echogenicity was detected in patients with volume depletion or obstructive nephropathy all of them complicated with infection or diabetes. Only five patients with AKI had increased RRI. The causes for increased RRI were: DKA with severe infection, sepsis with severe coronary heart disease, obstructive nephropathy caused by a metastatic malignant tumor, ATN, and LN secondary antiphospholipid antibody syndrome with lower extremity venous thrombosis. Although there were 12 cases with hydronephrosis, obstructive nephropathy was confirmed in only 8 cases. Two cases were considered to have physiological hydronephrosis. In two cases, AKI could not be fully explained by unilateral hydronephrosis.

There were no AKI cases caused due to renal artery 
Table 3 Abnormal findings of US evaluation in 111 patients with AKI

\begin{tabular}{|c|c|}
\hline Items & Total \\
\hline \multirow{7}{*}{$\begin{array}{l}\text { At least one kidney }>120 \mathrm{~mm} \\
\text { long }(\mathrm{N}=15)\end{array}$} & Diabetic nephropathy [2] \\
\hline & ATN [2] \\
\hline & Severe pancreatitis [1] \\
\hline & Sepsis [3] \\
\hline & Urinary retention with sepsis [1] \\
\hline & AAV [1] \\
\hline & Unilateral nephrolithiasis with sepsis [1] \\
\hline \multirow{8}{*}{$\begin{array}{l}\text { Increased parenchymal } \\
\text { echogenicity }(\mathrm{N}=26)\end{array}$} & IgA nephropathy [1], AAV [1], lupus nephritis [2], membranous nephropathy [3], nephrotic syndrome [4] \\
\hline & ATN [3] \\
\hline & DKA with pyelonephritis [1] \\
\hline & Sepsis [4] \\
\hline & $\mathrm{A} / \mathrm{C}[2]$ \\
\hline & Heart failure [2] \\
\hline & Volume depletion [2] \\
\hline & Obstructive nephropathy [1] \\
\hline Hydronephrosis $(\mathrm{N}=12)$ & Unilateral hydronephrosis [2] \\
\hline
\end{tabular}

US, ultrasonography; AKI, acute kidney injury; RRI, renal resistance index; ATN, acute tubular necrosis; AAV, antibody associated vasculitis; DKA, diabetic ketoacidosis; LN, lupus nephritis.

stenosis, congenital urinary system malformation, or renal vein thrombosis. The most common incidental findings of US evaluation were renal cysts, including 8 unilateral and 11 bilateral renal cysts. A renal mass was detected in one patient.

Among the 111 patients with AKI, 56 patients also received US evaluation of the liver. Of these patients, 21 were diagnosed by US as having steatosis. Two patients with increased parenchymal echogenicity also had steatosis.

\section{Discussion}

In the present study, only $34.23 \%$ [38] of the 111 AKI patients had increased parenchymal echogenicity, hydronephrosis, or increased RRI. Renal cysts were the most common accidental finding. Compared with the control group, the kidneys of patients with AKI were significantly larger.

Although a majority of the patients in this study had 
"normal kidneys" according to US evaluation, kidney size and parenchymal thickness were significantly greater in AKI patients than in the control group. With increasing age, parenchymal reduction leads to decreased renal size (11). In Emamian et al.'s study the median length of the left and right kidneys was 112 and $109 \mathrm{~mm}$, respectively (11). In another large-scale investigation (16), thin-layer CT scan was performed to evaluate 2068 kidneys in 1,040 adults. The average length of the left and right kidneys was $111.3 \pm 12.6$ and $108.5 \pm 12.2 \mathrm{~mm}$, respectively. Many factors were found to affect kidney size, the most important of which included body mass index, height, gender, age, kidney position, and renal artery stenosis (16).

Kidney length is the most common index for evaluating the size of a kidney, but kidney volume rather than kidney length is regarded as a valuable predictor. Renal volume is correlated with endogenous creatinine clearance (17). In Emamian et al.'s study (11), the median volume was $146 \mathrm{~cm}^{3}$ for the left kidney and $134 \mathrm{~cm}^{3}$ for the right kidney. In the present study, the average kidney size was slightly smaller than those recorded in previous studies. However, the subjects included in Emamian (11) and Glodny's (16) studies were from Denmark and Australia, respectively, and race and height might affect kidney size. Although the kidney size of the AKI patients included in this study was significantly larger than that of the control group, the length of the kidney in most of the patients ranged between 90 and $120 \mathrm{~mm}$. It is difficult to determine enlargement in kidney based on a single US evaluation. Repeated US evaluation might provide useful information for assessing AKI.

Parenchymal thickness also acts as a useful clinical maker in kidney evaluation. Although previous research has indicated the renal parenchyma to normally be $15-16 \mathrm{~mm}$ thick, a standard thickness has not yet been agreed (11). In Mounier-Vehier et al.'s study (18). the kidneys of 49 patients with hypertension were evaluated by spiral CT. Although the size of the kidneys was still within the normal range, the thickness of the cortex after renal artery stenosis was reduced. In the present study, the renal parenchyma was slightly thicker in the AKI group than in the control group, and the right kidney showed a significant difference.

Renal US evaluation also acts as an important indicator by which to differentiate AKI and CKD. Although most of the patients with AKI and CKD can be differentiated by their past medical history, the medical history of some patients is uncertain. During the evaluation of renal US, thinner parenchyma with increased echogenicity has been used as an indication of irreversible CKD, thus avoiding further unnecessary assessments, biopsies, and therapies $(1,4)$. Normal kidney size is difficult to define. Emamian et al. suggested that kidneys are normally in the range of 100$120 \mathrm{~mm}$ (11). However, Glodny et al. proposed that kidney size can range widely and even a left kidney $<80 \mathrm{~mm}$ in length or $>140 \mathrm{~mm}$ in length can be considered normal (16). In the present study, 3 patients had bilateral kidneys $<90 \mathrm{~mm}$ in length, with the serum creatinine level decreased to $<88.4 \mu \mathrm{mol} / \mathrm{L}$ after recovery. The present study further supported the idea that a wider range should be applied for "normal" renal length.

The etiologies of AKI were investigated in 748 patients with acute renal failure in a multicenter prospective study (19). Only $3.3 \%$ of patients required intervention due to obstructive nephropathy (19). Podoll et al.'s retrospective study analyzed the data of 810 patients with AKI (6). Of these cases, 62\% [500] had "normal" kidneys based on US evaluation. The proportion of AKI attributed to obstructive nephropathy remained low $(6,19)$. In the present study, 12 out of 111 AKI patients evaluated by renal US had hydronephrosis. Of these 12 patients, 8 were diagnosed as having obstructive nephropathy. Only $7.21 \%$ of patients who underwent US evaluation required intervention due to obstructive nephropathy. In most cases, patients with obstructive nephropathy-associated AKI demonstrated significant clinical manifestations $(5,6)$. Only in rare cases has imaging formed the entire basis for the diagnosis of obstructive nephropathy $(5,6)$. For this reason, US has not been recommended as a routine evaluation for AKI by previous studies $(5,6)$. These studies also concluded that renal US cannot provide useful information related to AKI $(5,6)$. In the present study, 49.12\% [111] of 226 patients with AKI received renal US. Prerenal AKI is relatively common in clinical practice. If volume depletion is promptly addressed, renal function can be quickly recovered. At present, US is not mandatory evaluation for prerenal AKI $(5,6)$.

The echogenicity of the renal cortex is produced by the backscattering of the glomerulus, renal blood vessels, and renal tubules (20). Moghaz et al. analyzed the correlation between ultrasound parameters (such as kidney length, cortical echogenicity, and cortical and parenchymal thickness) and histopathological changes of glomerulosclerosis, renal tubular atrophy, interstitial fibrosis, and interstitial inflammation in 207 patients (4). Renal tubular atrophy and interstitial inflammation, rather than interstitial fibrosis, are considered as factors 
for determining cortical histopathological changes (4). This is because the glomerulus accounts for only $8 \%$ of cortical volume, and increased parenchymal echogenicity does not necessarily change in glomerular diseases (21). In AKI caused by glomerular diseases, increased parenchymal echogenicity might indicate interstitial inflammation or renal tubules atrophy (A/C possibility). The US manifestations of acute tubular necrosis vary. In ATN secondary to ischemia, the kidney often appears to be "normal" (21). When ATN occurs (i.e., if too much Tamm-Horsfall protein is present in the renal tubules), the pyramids and normal cortex appear isoechoic (22). With the progressive development of kidney failure, the cortex becomes more echogenic, which indicates the deterioration of kidney function (22). Although only a small number of AKI patients have increased parenchymal echogenicity, parenchymal echogenicity changes and histopathological changes are interlinked. The increased parenchymal echogenicity might attribute to interstitial inflammation, renal tubules atrophy, and excessive protein casts $(4,20,22)$. In combination with renal parenchyma thickness and parenchyma echogenicity, it may provide useful information and influence changes in clinical management.

In the present study, 33 patients had coexisting glomerular disease and 3 had chronic renal failure. Onethird of patients with glomerular diseases had increased parenchymal echogenicity. Four cases of AKI resulted from heart failure or volume depletion and were accompanied with changes in parenchymal echogenicity. Prerenal AKI showed kidney changes in kidney function. The changes in parenchymal echogenicity suggest that pre-renal factor might not be the only cause of AKI. Further potential causes of AKI should also be screened. In summary, changes in renal parenchymal echogenicity hold potential value for the clinical management of AKI.

In recent years, some studies have suggested that Doppler US has a certain clinical value in the management of AKI. In Zhi et al.'s prospective study (8), Doppler US was used to evaluate RRI and predict the occurrence of AKI within six hours following admission. The results indicated RRI to be a predictor of AKI [area under the curve (AUC) 0.772]. Other previous studies have also supported the use of RRI as a predictor of AKI $(8,23,24)$. RRI is considered to be superior to cystatin $\mathrm{C}$ and has the same predictive value as NGAL for AKI $(23,24)$. In CKD patients, RRI $>0.65$ is related to severe interstitial fibrosis, severe arteriosclerosis, and severe renal function damage (25). RRI is considered to be an indicator for the occurrence and development of kidney diseases generally $(26,27)$. However, RRI is influenced by multiple factors and is only one of many evaluation indexes (26-28).

In AKI of different etiology, the predictive value of RRI is different. The pathogenesis of AKI in patients with heart failure remains complex. A reduction in cardiac output decreases renal blood flow and leads to renal ischemia $(8,27)$. RRI might be a predictor of AKI in patients with heart failure $(8,27)$. However, when AKI is caused by sepsis, the pathophysiological changes are much more complicated. Inflammatory factors with toxic effects are associated with the occurrence of AKI $(8,27)$. In these cases, RRI does not serve as a good predictor of AKI $(8,27)$. In cases with hydronephrosis and renal vein thrombosis, the increased pressure in the interstitium and veins changes the level of RRI (27). At this time, RRI is regarded as a reliable indicator for the severity of renal damage (27). In patients undergoing kidney transplants and those with CKD, increased RRI can be used as an independent predictor of kidney and clinical outcomes (27). In the present study, only five patients had increased RRI complications. As RRI in some patients was described only to be in the normal range, with no further details recorded, the correlation between RRI value and other clinical or ultrasound indicators was not analyzed. The application value of RRI in AKI should be further evaluated.

In the present study, more than $50 \%$ of AKI patients did not undergo US evaluation. Most patients who underwent evaluation by US were within the normal range. In the present study, apart from obstructive nephropathy, some patients had abnormal US findings, including increased parenchymal echogenicity and RRI. In addition, the present study indicated the AKI patients to have significantly larger kidneys than the control group. Moghaz et al. proposed the combination of renal size and parenchymal echogenicity for evaluating kidney disease (4). Previous studies have acknowledged the potential value of RRI for AKI (26-28). These may support the idea that, aside from its ability to exclude obstructive nephropathy, the use of US might hold further value.

However, our study had some limitations. Firstly, this was a single-center retrospective study, which made bias inevitable. Secondly, US was performed by multiple radiologists and the accuracy of US is highly dependent on the skills and experience of the operator. Although the radiologists at our hospital, have all received complete training and assessment, the measurement error could not be eliminated. Thirdly, the sample size and the number of 
cases with these malformations are small. Conclusion needs further verification. Finally, retrospective analysis of the etiologies of AKI can lead to inaccuracy.

\section{Conclusions}

In current clinical practice, US evaluation is not considered as a routine approach to AKI. Although most of patients with AKI are within the normal range, as evaluated by US, some useful findings other than obstruction can still be detected by US. Aside from its ability to exclude obstructive nephropathy, US evaluation might hold further value. As the kidney size of the AKI patients in this study was significantly larger than that of the healthy individuals, combining kidney size with other ultrasound indicators might have potential value in the evaluation of AKI.

\section{Acknowledgments}

Funding: None.

\section{Footnote}

Data Sharing Statement: Available at http://dx.doi. org/10.21037/tau-20-831

Conflicts of Interest: Both authors have completed the ICMJE uniform disclosure form (available at http://dx.doi. org/10.21037/tau-20-831). The authors have no conflicts of interest to declare

Ethical Statement: The authors are accountable for all aspects of the work in ensuring that questions related to the accuracy or integrity of any part of the work are appropriately investigated and resolved. This study strictly adhered to the guidelines of the Declaration of Helsinki of the World Medical Association. This retrospective study was approved by the ethics committee of The First affiliated Hospital of Jinzhou Medical University (File number: 202003). The ethics committee of the hospital waived the need for written informed consent from the patients due to the study's retrospective nature.

Open Access Statement: This is an Open Access article distributed in accordance with the Creative Commons Attribution-NonCommercial-NoDerivs 4.0 International License (CC BY-NC-ND 4.0), which permits the noncommercial replication and distribution of the article with the strict proviso that no changes or edits are made and the original work is properly cited (including links to both the formal publication through the relevant DOI and the license). See: https://creativecommons.org/licenses/by-nc-nd/4.0/.

\section{References}

1. O'Neill WC. Sonographic evaluation of renal failure. Am J Kidney Dis 2000,35:1021-38.

2. Rosenfield AT, Taylor KJ, Crade M, et al. Anatomy and pathology of the kidney by gray scale ultrasound. Radiology 1978,128:737-44.

3. Hricak H, Cruz C, Romanski R, et al. Renal parenchymal dis-ease: Sonographic-histologic correlation. Radiology 1982;144:141-7.

4. Moghazi S, Jones E, Schroepple J, et al. Correlation of renal histopathology with sonographic findings. Kidney Int 2005;67:1515-20.

5. Niu XL, Wu Y, Hao S, et al. Value of micro-proteinuria in combination with ultrasonography of the left renal vein in the diagnosis of orthostatic proteinuria. Ann Transl Med 2019;7:780.

6. Podoll A, Walther C, Finkel K. Clinical utility of gray scale renal ultrasound in acute kidney injury. BMC Nephrology 2013;14:188.

7. Capotondo L, Nicolai GA, Garosi G. The role of color Doppler in acute kidney injury. Arch Ital Urol Androl 2010;82:275-9.

8. Zhi HJ, Zhao J, Nie S, et al. Semiquantitative Power Doppler Ultrasound Score to Predict Acute Kidney Injury in Patients With Sepsis or Cardiac Failure: A Prospective Observational Study. J Intensive Care Med 2019;885066619887333.

9. Venkatachalam MA, Weinberg JM, Kriz W, et al. Failed Tubule Recovery, AKI-CKD Transition, and Kidney Disease Progression. J Am Soc Nephrol 2015;26:1765-76.

10. Available online: http://kdigo.org/home/guidelines/acutekidney-injury/

11. Emamian SA, Nielsen MB, Pedersen JF, et al. Kidney dimensions at sonography: correlation with age, sex, and habitus in 665 adult volunteers. AJR Am J Roentgenol 1993;160:83-6.

12. Hricak H, Lieto RP. Sonographic determination of renal volume. Radiology 1983;148:311-2.

13. Vade A, Lau P, Smick J, et al. Sonographic renal parameters as related to age. Pediatr Radiol 1987;17:212-5.

14. Platt JF, Rubin JM, Bowerman RA, et al. The inability to detect kidney disease on the basis of echogenicity. AJR Am 
J Roentgenol 1988;151:317-9.

15. Boddi M, Natucci F, Cian E. The internist and the renal resistive index: truths and doubts. Intern Emerg Med 2015;10:893-905

16. Glodny B, Unterholzner V, Taferner B, et al. Normal kidney size and its influencing factors - a 64-slice MDCT study of 1.040 asymptomatic patients. BMC Urol 2009,9:19.

17. Sanusi AA, Arogundade FA, Famurewa OC, et al. Relationship of ultrasonographically determined kidney volume with measured GFR, calculated creatinine clearance and other parameters in chronic kidney disease (CKD). Nephrol Dial Transplant 2009;24:1690-4

18. Mounier-Vehier C, Lions C, Devos P, et al. Cortical thickness: an early morphological marker of atherosclerotic renal disease. Kidney Int 2002;61:591-8.

19. Liaño F, Pascual J. Epidemiology of acute renal failure: a prospective, multicenter, community-based study. Madrid Acute Renal Failure Study Group. Kidney Int 1996;50:811-8.

20. Insana MF, Hall TJ, Fishback JL. Identifying acoustic scattering sources in normal renal parenchyma from the anisotropy in acoustic properties. Ultrasound Med Biol 1991;17:613-26.

21. Quaia E, Bertolotto M. Renal parenchymal disease: Is

Cite this article as: Liu C, Wang X. Clinical utility of ultrasonographic evaluation in acute kidney injury. Transl Androl Urol 2020;9(3):1345-1355. doi:10.21037/tau-20-831 characterization feasible with ultrasound? Eur Radiol 2002;12:2006-20.

22. Khati NJ, Hill MC, Kimmel, PL. The role of ultrasound in renal insufficiency: the essentials. Ultrasound $\mathrm{Q}$ 2005;21:227-44.

23. Lubas A, Kade G, Niemczyk S. Renal resistive index as a marker of vascular damage in cardiovascular diseases. Int Urol Nephrol 2014,46:395-402.

24. Schnell D, Deruddre S, Harrois A, et al. Renal resistive index better predicts the occurrence of acute kidney injury than cystatin C. Shock 2012,38:592-7.

25. Bigé N, Lévy PP, Callard P, et al. Renal arterial resistive index is associated with severe histological changes and poor renal outcome during chronic kidney disease. BMC Nephrol 2012;13:139.

26. Doi Y, Iwashima Y, Yoshihara F, et al. Association of renal resistive index with target organ damage in essential hypertension. Am J Hypertens 2012;25:1292-8.

27. Boddi M, Natucci F, Ciani E. The internist and the renal resistive index: truths and doubts. Intern Emerg Med 2015;10:893-905.

28. Boddi M. Renal Ultrasound (and Doppler Sonography) in Hypertension: An Update. Adv Exp Med Biol 2017;956:191-208. 


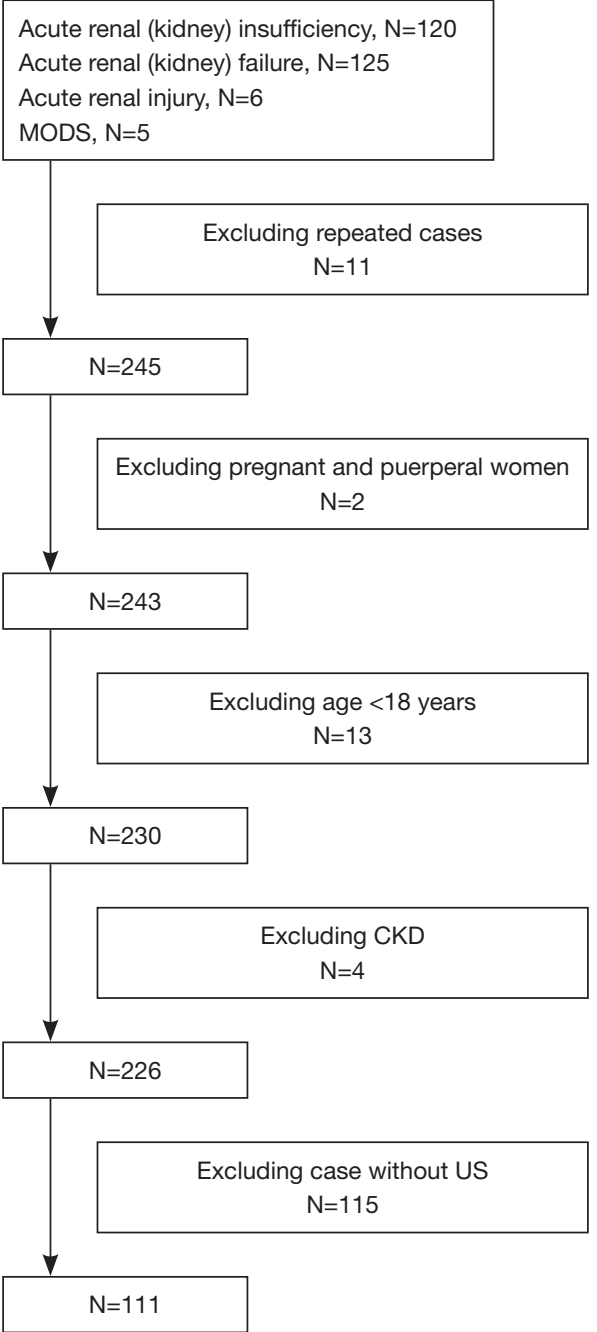

Figure S1 Flow chartof this study. MODS, multiple organ dysfunction syndrome; CKD, chronic kidney disease; US, ultrasonography. 